Regular Article

\title{
Silver chloride enwrapped silver grafted on nitrogen-doped reduced graphene oxide as a highly efficient visible-light-driven photocatalyst
}

\author{
Liang Wang ${ }^{\mathrm{a}, \mathrm{b}}$, Yilun Shi ${ }^{\mathrm{a}, \mathrm{b}}$, Tianfu Wang ${ }^{\mathrm{c}}$, Lili Zhang ${ }^{\mathrm{d}, *}$ \\ a State Key Laboratory of Separation Membranes and Membrane Processes, Tianjin Polytechnic University, Tianjin 300387, China \\ ${ }^{\mathrm{b}}$ School of Environmental and Chemical Engineering, Tianjin Polytechnic University, Tianjin 300387, China \\ ${ }^{\mathrm{c}}$ Tsinghua University High School, Beijing 100085, China \\ ${ }^{\mathrm{d}}$ Key Laboratory of Drinking Water Science and Technology, Research Center for Eco-Environmental Sciences, Chinese Academy of Sciences, Beijing 100085, China
}

G R A P H I C A L A B S T R A C T
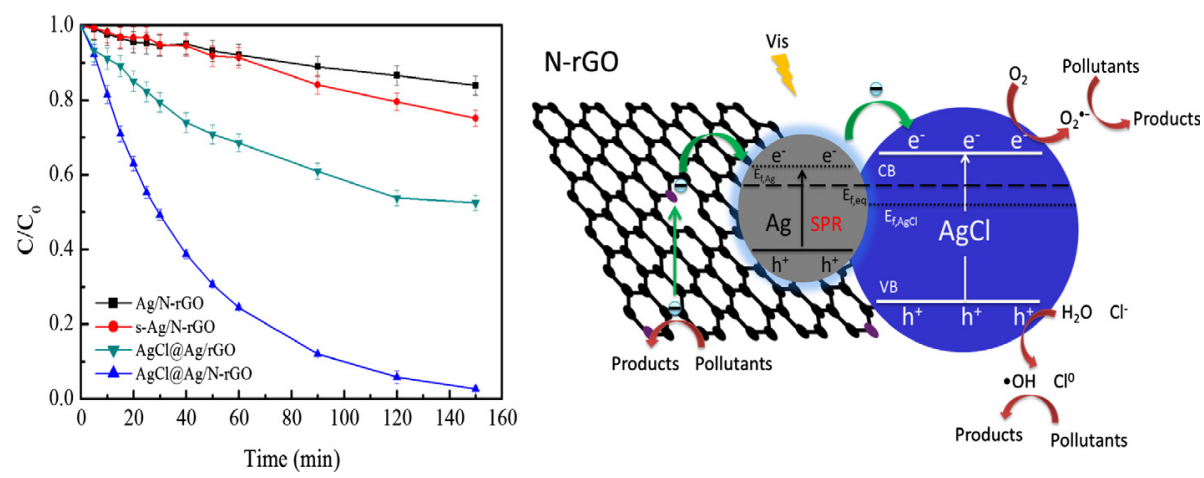

\section{A R T I C L E I N F O}

\section{Article history:}

Received 18 April 2017

Revised 10 June 2017

Accepted 12 June 2017

Available online 13 June 2017

\section{Keywords:}

$\mathrm{AgCl} @ \mathrm{Ag} / \mathrm{N}-\mathrm{rGO}$

Interfacial contact

Photocatalyst

Visible light

\begin{abstract}
A B S T R A C T
The visible-light-driven plasmonic photocatalyst silver chloride enwrapped silver/nitrogen-doped reduced graphene oxide $(\mathrm{AgCl} @ \mathrm{Ag} / \mathrm{N}-\mathrm{rGO})$ was prepared by a facile hydrothermal-in situ oxidation method and characterized by Scanning electron microscopy (SEM), X-ray diffraction (XRD), Fourier transform-infrared spectroscopy (FTIR), Raman spectroscopy, X-ray photoelectron spectroscopy (XPS) and UV-vis diffuse reflectance spectroscopy (UV-vis DRS). The characterization results reveal that Ag nanoparticles (NPs) were first grafted on N-rGO via N-groups as anchor sites and then enwrapped by $\mathrm{AgCl}$ by in situ oxidation. Close interfacial contact favors efficient electron transfer, leading to high photoactivity and photostability for the degradation of various toxic organic pollutants. The photocatalytic performance of this photocatalyst was significantly higher than that of $\mathrm{AgCl} @ \mathrm{Ag} / \mathrm{rGO}$ and other related photocatalysts due to the in situ introduction of N-groups. Additionally, the used catalyst can be recycled without an appreciable loss of catalytic activity. Based on electron spin resonance and cyclic voltammetry analyses, the electron transfer processes were confirmed to occur from plasmon-induced Ag NPs to AgCl and from N-rGO to Ag NPs, and pollutants could be oxidized through the loss of electrons to N-rGO by the interaction between the pollutants and N-rGO. The active species of superoxide anion radicals $\left(\mathrm{O}_{2}{ }^{-}{ }^{-}\right)$, photogenerated holes $\left(\mathrm{h}^{+}\right)$and surface-adsorbed $\cdot \mathrm{OH}$ played roles in pollutant photodegradation. Accordingly, the plasmon-induced electron transfer processes elucidated photostability of AgCl@Ag/N-rGO.AgCl@Ag/ $\mathrm{N}-\mathrm{rGO}$ has a potential application in water purification due to its high photoactivity and photostability.
\end{abstract}

(c) 2017 Elsevier Inc. All rights reserved.

\footnotetext{
* Corresponding author.

E-mail address: llzhang@rcees.ac.cn (L. Zhang).
} 


\section{Introduction}

With the rapid growth of worldwide industrialization, severe environmental problems have become a major concern $[1,2]$. Photocatalysis is an attractive approach for solving environmental issues using solar energy conversion $[3,4]$. The most widely investigated photocatalyst is $\mathrm{TiO}_{2}$ owing to its low cost and structural stability. Unfortunately, due to the wide band gap of $\mathrm{TiO}_{2}$, it is only activated under ultraviolet irradiation [5]. For solar energy utilization, the search for visible-light-driven photocatalysts is still one of the most challenging tasks.

Among the various types of visible-light-driven photocatalysts, plasmonic photocatalysts comprising metal nanoparticles (NPs) (such as $\mathrm{Au}, \mathrm{Pt}$, and $\mathrm{Ag}$ ) have attracted particular attention in the past several years due to the surface plasmon resonance (SPR) effect of the metal NPs in the visible region [6,7]. More specifically, silver/silver halide-based $(\mathrm{Ag} / \mathrm{AgX}, \mathrm{X}=\mathrm{Br}, \mathrm{Cl})$ nanomaterials have been reported for their excellent photocatalytic performances in the degradation of pollutants under visible-light irradiation [8,9]. For example, several $\mathrm{Ag} / \mathrm{AgX}$-based photocatalysts, including $\mathrm{Ag} /$ $\mathrm{AgCl}$ [10], Ag@AgBr [11] and Ag-AgI [12] have been developed. The high photocatalytic activity of these catalysts is mainly ascribed to the SPR effect of the Ag NPs and the interaction between $\mathrm{Ag}$ and $\mathrm{AgX}$. However, the low surface area of pure $\mathrm{Ag} /$ $\mathrm{AgX}$ and the recombination of plasmon-induced electron-hole pairs before arriving at the surface lead to a loss of plasmonic photoactivity [13]. Therefore, it is meaningful to synthesize a new hybrid material that can enhance the photocatalytic activity and stability through increasing the surface of area and promoting the charge transfer.

Reduced graphene oxide ( $\mathrm{rGO}$ ), a unique $\mathrm{sp}^{2}$ hybrid carbon network material, has received considerable attention owing to its extremely high surface area, high thermal and chemical stability, and excellent charge carrier mobility [14]. To enhance the specific capacity of graphene, chemical doping of graphene, such as B [15], $\mathrm{N}$ [16] and organic molecules [17], has been investigated. Among them, $\mathrm{N}$-doped reduced graphene oxide $(\mathrm{N}-\mathrm{rGO})$ was found to aid in tailoring its electronic properties and improving its photocatalytic efficiency [18]. Three typical $\mathrm{N}$-bonds quaternary $\mathrm{N}$, pyridinic $\mathrm{N}$ and pyrrolic $\mathrm{N}$, in $\mathrm{N}-\mathrm{rGO}$ have been widely reported and their effects on the photocatalytic performance have also been investigated [19]. Compared with pristine graphene, $\mathrm{N}$-doped graphene has more activated regions, which can anchor metals [20] or metal oxides [21] on the reduced graphene materials. For example, Marcilla et al. [22] reported $\mathrm{NiCoMnO}_{4}$ nanoparticles anchored on nitrogen-doped graphene nanosheets as highly efficient bifunctional electrocatalysts for oxygen reduction evolution reactions. Niu et al. [23] constructed $\mathrm{AgBr}$ nanoparticles supported on a $\mathrm{g}-\mathrm{C}_{3} \mathrm{~N}_{4}$-decorated nitrogendoped graphene nanocomposite, which demonstrated a high efficiency for organic contaminant degradation and $\mathrm{CO}_{2}$ reduction under visible light. Therefore, plasmonic photocatalysts anchored on nitrogen-doped graphene nanosheets could be more efficient and stable for wastewater treatment. Moreover, Quan et al. [24] synthesized a plasmonic photocatalyst $\mathrm{Ag} @ \mathrm{AgCl} / \mathrm{RGO}$ through a precipitation reaction followed by photoreduction, which exhibited an excellent photocatalytic activity. Wei et al. [25] prepared nitrogendoped grapheme-modified AgX@Ag (NG-AgX@Ag, X = Br, Cl) by a co-precipitation method, which showed a greatly improved photocatalytic activity compared to AgX@Ag. These photocatalysts were constructed by compositing individual components. Due to close interfacial interactions that efficiently promote charge transfer [26], it is necessary to develop an in situ convenient way to prepare the effective composite photocatalysts.

In this study, we anchored AgCl@Ag on N-rGO by a facile hydrothermal-in situ oxidation method. The obtained composites showed distinctly enhanced photocatalytic activities and stabilities for the degradation of pollutants, including 2-chlorophenol (2-CP), bisphenol A (BPA), phenol, and 2,4-dichlorophenoxyacetic acid (2,4-D) under visible light irradiation, resulting from the SPR of the Ag NPs and the anchoring effect between the Ag NPs and the $\mathrm{N}-\mathrm{rGO}$. The possible mechanism for the photocatalytic system was investigated.

\section{Experiment}

\subsection{Chemicals}

Silver nitrate $\left(\mathrm{AgNO}_{3}\right)$, Iron (III) chloride hexahydrate $\left(\mathrm{FeCl}_{3}-\right.$ $6 \mathrm{H}_{2} \mathrm{O}$ ) and phenol were purchased from Sinopharm Chemical Reagent Co., Ltd. BPA, 2,4-D, diphenhydramine (DP), and phenytoin (PHT) were obtained from Acros (Geel, Belgium). 2-CP was purchased from Sigma-Aldrich (St Louis, United States). 5-tert-Butoxy carbonyl-5-methyl-1-pyrroline-N-oxide (BMPO) was supplied by the Bioanalytical Lab (Sarasota, FL). All chemicals were at least analytical grade. The molecular structures of 2-CP, phenol, BPA, 2,4-D, DP and PHT are shown in Fig. S1. Deionized water was used throughout this study.

\subsection{Preparation}

A graphene oxide (GO) dispersion in water was prepared by sonication of graphite oxide that was obtained via a chemical exfoliation of natural graphite following the modified Hummers method [27]. An Ag/N-rGO nanocomposite was synthesized by a hydrothermal method. Typically, $60 \mathrm{~mL}$ GO solution (0.05 wt\%) and $100 \mathrm{~mL}$ silver ammonia solution $\left(0.1 \mathrm{M} \mathrm{AgNO}_{3}\right.$ in $3 \mathrm{M} \mathrm{NH}_{3}$ $\mathrm{H}_{2} \mathrm{O}$ ) were mixed and hydrothermally treated at $130{ }^{\circ} \mathrm{C}$ for $3 \mathrm{~h}$. After cooling down to room temperature, the resulting samples were centrifuged, washed and dried in vacuum at $60{ }^{\circ} \mathrm{C}$ for $5 \mathrm{~h}$. Following this procedure, $\mathrm{Ag} / \mathrm{N}-\mathrm{rGO}(x \mathrm{wt} \%)$ was obtained, where $x$ represents the weight ratios of GO to $\mathrm{Ag}$ in the preparation process. $\mathrm{N}-\mathrm{rGO}$ was synthesized through the same steps used for making $\mathrm{Ag} / \mathrm{N}-\mathrm{rGO}$ without adding $\mathrm{AgNO}_{3}$, and $\mathrm{Ag} / \mathrm{rGO}$ was prepared without adding $\mathrm{NH}_{3} \mathrm{H}_{2} \mathrm{O}$.

The AgCl@Ag/N-rGO nanocomposite was prepared by an in situ oxidation reaction from $\mathrm{Ag} / \mathrm{N}-\mathrm{rGO}$ and $\mathrm{FeCl}_{3}$ at room temperature. Typically, $0.5 \mathrm{~g} \mathrm{Ag} / \mathrm{N}-\mathrm{rGO}$ ( $x$ wt\%) was added into $40 \mathrm{~mL}$ of a $0.1 \mathrm{M}$ $\mathrm{FeCl}_{3}$ aqueous solution and stirred for $30 \mathrm{~min}$. Then, the solid sample was quickly centrifuged, washed and dried in vacuum at $60{ }^{\circ} \mathrm{C}$ for $5 \mathrm{~h}$. The synthesized samples were designated as $\mathrm{AgCl} @ \mathrm{Ag} / \mathrm{N}$ rGO ( $x$ wt\%, y), where y indicates the volume of $\mathrm{FeCl}_{3}$ added in the preparation process. The AgCl@Ag/N-rGO (3 wt\%, 40) photocatalyst exhibited the highest photocatalytic activity, stability and crystallinity, as shown in Figs. S2-S5 and Table S1. This catalyst was used for all of the subsequent experiments unless otherwise specified. As references, s-Ag/N-rGO (3 wt\%) was prepared, as described above, with $\mathrm{Fe}\left(\mathrm{NO}_{3}\right)_{3}$ added instead of $\mathrm{FeCl}$. $\mathrm{AgCl} @ \mathrm{Ag} /$ rGO (3 wt\%, 40) was prepared by an in situ oxidation reaction from $\mathrm{Ag} / \mathrm{rGO}$ and $\mathrm{FeCl}_{3}$ at room temperature, as described above.

\subsection{Characterization}

The powder X-ray diffraction (XRD) patterns of the samples were recorded on a Scintag-XDS-2000 diffractometer with $\mathrm{Cu} \mathrm{K \alpha}$ radiation ( $\mathrm{k}=1.540598 \AA$ ) at $40 \mathrm{kV}$ and $40 \mathrm{~mA}$. The X-ray photoelectron spectroscopy (XPS) data were taken on a Kratos AxisUltra instrument with monochromatic $\mathrm{Al} \mathrm{K \alpha}$ radiation $(225 \mathrm{~W}$, $15 \mathrm{~mA}, 15 \mathrm{kV}$ ). The $\mathrm{C} 1 \mathrm{~s}$ photoelectron binding energy was set at $284.8 \mathrm{eV}$ and used as reference for calibrating other peak positions. The UV-vis diffuse reflectance spectra (UV-vis DRS) were con- 
ducted on a Hitachi U-3900 spectrophotometer with an integrating sphere attachment. Fourier transform-infrared (FTIR) spectra were acquired using a Bruker FTIR. Raman spectra were recorded on a LabRAM HR Evolution (HORIBA, France) equipped with a CCD detector using a laser source at an excitation line of $532 \mathrm{~nm}$. The field emission scanning electron microscope (FESEM) and energy dispersive X-ray spectrometer (EDS) were performed using an SU8020 FESEM instrument (Hitachi), which was operated at the accelerating voltage of $20 \mathrm{kV}$ and a detector current of $10 \mathrm{~mA}$. The electron spin resonance (ESR) spectra were obtained with a Bruker A300-10/12 ESR spectrometer using BMPO as a spin trap agent to detect reactive oxygen species. The photoelectrochemical measurements were performed on a $\mathrm{CHI}$ 660D electrochemical workstation with a standard three-electrode system. The prepared photocatalyst electrode was employed as the working electrode, a Pt wire and saturated calomel electrode (SCE) served as the counter electrode and reference electrode, and a $0.1 \mathrm{M} \mathrm{Na}_{2} \mathrm{SO}_{4}$ solution was used as the electrolyte.

\subsection{Photocatalytic activity}

The photocatalytic activity of the AgCl@Ag/N-rGO was evaluated by the degradation of 2-CP, BPA, phenol, 2,4-D, DP and PHT under visible light irradiation. A $150 \mathrm{~W}$ Xe arc lamp with a 400$\mathrm{nm}$ cutoff glass filter was used as the light source. The incident visible light intensity was measured with a radiometer (Photoelectric Instrument Factory Beijing Normal University), and the intensity was $3.5 \mathrm{~mW} \mathrm{~cm}^{-2}$. Prior to illumination, $60 \mathrm{~mL}$ of a suspension containing $1.6 \mathrm{~g} \mathrm{~L}^{-1}$ photocatalysts and $10 \mathrm{mg} \mathrm{L}^{-1}$ pollutant solution was vigorously stirred in the dark for $30 \mathrm{~min}$ to establish the adsorption-desorption equilibrium. During irradiation, the suspension samples were withdrawn at given time intervals and filtered to remove the catalyst powders for analysis. The concentrations of the organic pollutants were measured using a highperformance liquid chromatograph (Agilent 1200 series, U.S.A.) equipped with a UV detector and a Zorbax SB-Aq column $(5 \mu \mathrm{m}$, $4.6 \times 250 \mathrm{~mm}$; Agilent). The mobile phase consisted of a mixture of methanol/water or a mixture of acetonitrile/water (with $0.08 \%$ phosphoric acid) at a flow rate of $1.0 \mathrm{~mL} \mathrm{~min}^{-1}$. The total organic carbon (TOC) of the solution was analyzed using a TOC- $\mathrm{V}_{\mathrm{CPH}}$ analyzer (Shimadzu). The released metallic ions from the catalysts in the reaction process were determined by ICP-OES on an OPTIMA 2000 (Perkin Elmer Co., USA). To test the stability and recyclability of $\mathrm{AgCl} @ \mathrm{Ag} / \mathrm{N}-\mathrm{rGO}$, the catalyst was filtered, washed and dried at $60{ }^{\circ} \mathrm{C}$ in vacuum. The catalyst used again in the second cycle. This process was repeated several times.

All the reported data were the average of the triplicates with a standard deviation of less than $5 \%$.

\section{Results and discussion}

\subsection{Materials characterization}

Fig. $1 \mathrm{~A}$ and $\mathrm{B}$ show the FESEM image of N-rGO and AgCl@Ag/NrGO, respectively. $\mathrm{N}-\mathrm{rGO}$ showed a transparent, thin and wrinkled morphology, which displayed the typical graphene structure. It is clear that the AgCl@Ag particles were enwrapped by N-rGO, indicating that the AgCl@Ag particles attached onto the N-rGO sheets [28]. Meanwhile, the EDS analysis (inset in Fig. 1B) and elemental mapping (Fig. 1C) indicate the existence of $\mathrm{C}, \mathrm{N}, \mathrm{Cl}$ and $\mathrm{Ag}$ in $\mathrm{AgCl} @ \mathrm{Ag} / \mathrm{N}-\mathrm{rGO}$. The concentrations of the nitrogen, silver and chlorine were 8.71 at.\%, 25.05 at.\% and 20.17 at.\% from the EDS analysis. The atomic ratio of silver and chlorine was approximately 1.24 .
The typical XRD patterns of GO, N-rGO, Ag/N-rGO and AgCl@Ag/ $\mathrm{N}-\mathrm{rGO}$ are shown in Fig. 2. GO showed a sharp peak at $2 \theta=12.1^{\circ}$, corresponding to an interlayer d-spacing of $0.73 \mathrm{~nm}$ between the stacked graphite oxide sheets [29]. After the reduction with the ammonia solution by a hydrothermal method, the sharp diffraction peak of GO disappeared while a weak and broad diffraction peak at $2 \theta=23.9^{\circ}$ with an interlayer $\mathrm{d}$-spacing of $0.37 \mathrm{~nm}$ appeared in $\mathrm{N}$ rGO, indicating the efficient reduction of GO [29]. The reduction of GO is further demonstrated by the FTIR spectra in Fig. 3. The broad peak at $3371 \mathrm{~cm}^{-1}$ ascribed to the strong absorption of hydroxyl groups on the GO surface after the reduction with ammonia solution the hydroxyl groups almost disappeared in the spectra of $\mathrm{N}-\mathrm{rGO}, \mathrm{Ag} / \mathrm{N}-\mathrm{rGO}$ and $\mathrm{AgCl} @ \mathrm{Ag} / \mathrm{N}-\mathrm{rGO}$. The same is true for the oxygen-containing functional groups at $1728 \mathrm{~cm}^{-1} \quad(\mathrm{C}=\mathrm{O})$, $1225 \mathrm{~cm}^{-1}(\mathrm{C}-\mathrm{OH})$ and $1053 \mathrm{~cm}^{-1}(\mathrm{C}-\mathrm{O})$ [30]. However, for the spectra of $\mathrm{Ag} / \mathrm{rGO}$ and $\mathrm{AgCl} @ \mathrm{Ag} / \mathrm{rGO}$, the oxygen-containing functional groups decreased, but still existed, indicating that the ammonia solution could promote the reduction of GO. On the XRD diffractogram of $\mathrm{Ag} / \mathrm{N}-\mathrm{rGO}$ (Fig. 2), five characteristic peaks at approximately $38^{\circ}, 44^{\circ}, 64^{\circ}, 77^{\circ}$ and $84^{\circ}$ represented the (111), (200), (220), (311) and (222) planes of metallic Ag (JCPDS 65-2871). For AgCl@Ag/N-rGO, the diffraction peaks corresponding to the $\mathrm{AgCl}$ phase (JCPDS 85-1355) could be clearly observed, and the corresponding diffraction peaks of metallic Ag decreased significantly. Due to the relatively low content of $\mathrm{N}-\mathrm{rGO}$ in the $\mathrm{Ag}$ / $\mathrm{N}-\mathrm{rGO}$ and $\mathrm{AgCl} @ \mathrm{Ag} / \mathrm{N}-\mathrm{rGO}$, negligible diffraction peaks ascribed to N-rGO could be detected by XRD. Compared to the XRD pattern of AgCl@Ag/N-rGO, AgCl@Ag/rGO showed relatively low diffraction intensity (Fig. S6). That was due to the much smaller crystallite sizes and higher crystallinity of AgCl@Ag/N-rGO. The Raman spectra of the different samples are shown in Fig. 4. Two characteristic peaks at approximately $1355 \mathrm{~cm}^{-1}$ and $1600 \mathrm{~cm}^{-1}$ corresponding to the $\mathrm{D}$ band and $\mathrm{G}$ band were observed in $\mathrm{Ag} / \mathrm{N}-\mathrm{rGO}$. The $\mathrm{D}$ band is attributed to the vibrations of carbon atoms with dangling bonds in disordered graphite planes and the defects [31]. The G band corresponds to the $E_{2 g}$ vibration mode arising from the in-plane bond stretching of the $\mathrm{C}-\mathrm{C} \mathrm{sp^{2 }}$ bond [32]. The intensity ratio of the $\mathrm{D}$ band to the $G$ band refers to the degree of defects (or the defect density) in the carbon materials [33]. The ratio of the $\mathrm{D}$ band and $G$ band intensities $\left(\mathrm{I}_{\mathrm{D}} / \mathrm{I}_{\mathrm{G}}\right)$ of $\mathrm{Ag} / \mathrm{N}-\mathrm{rGO}$ (1.26) was significantly higher than that of GO (0.81) and slightly higher than that of NrGO (1.09), indicating the reduction of oxygen-containing groups in $\mathrm{GO}$ and the existence of more defects in the $\mathrm{N}-\mathrm{rGO}$ of $\mathrm{Ag} / \mathrm{N}$ rGO [34]. After in situ oxidation, the $\mathrm{I}_{\mathrm{D}} / \mathrm{I}_{\mathrm{G}}$ of $\mathrm{AgCl} @ \mathrm{Ag} / \mathrm{N}-\mathrm{rGO}$ (1.00) decreased compared to that of $\mathrm{Ag} / \mathrm{N}-\mathrm{rGO}$, indicating the partial re-oxidation of the defects by $\mathrm{Fe}^{3+}$ ions [35]; meanwhile, it was still higher than that of GO, indicating some of the defects in $\mathrm{N}-\mathrm{rGO}$ were well maintained [36].

To further verify the structure of AgCl@Ag/N-rGO, the surface chemical composition and chemical states were characterized by XPS (Fig. 5). As shown in the inset of Fig. 5, the N1s spectrum could be deconvoluted into two components: the pyridinic nitrogen $(\mathrm{N}$ 6) at $399 \mathrm{eV}$ and the pyrrolic nitrogen $(\mathrm{N}-5)$ at $400 \mathrm{eV}$. Specifically, pyridinic $\mathrm{N}(\mathrm{N}-6)$ bonded with two carbon atoms at the edges or defects of graphene and donated one $\mathrm{p}$ electron to the $\pi$ system; meanwhile, pyrrolic $\mathrm{N}(\mathrm{N}-5)$ connected with two carbon atoms and contributed two electrons to the system [37]. The results confirm that nitrogen atoms were introduced. The N-groups on rGO could serve as favorable nucleation and anchor sites for Ag NPs owing to the coordination with $\mathrm{Ag}^{+}$[21]; thus, a strong coupling between $\mathrm{Ag}$ and $\mathrm{N}-\mathrm{rGO}$ existed in $\mathrm{AgCl} @ \mathrm{Ag} / \mathrm{N}-\mathrm{rGO}$. The two peaks located at approximately $367.4 \mathrm{eV}$ and approximately $373.4 \mathrm{eV}$ in the $\mathrm{Ag}$ 3d spectra (Fig. S7A) were ascribed to the $\mathrm{Ag} 3 \mathrm{~d}_{5 / 2}$ and $\mathrm{Ag}$ $3 \mathrm{~d}_{3 / 2}$ binding energies, respectively. Furthermore, the peaks of $\mathrm{Ag}$ $3 d_{5 / 2}$ and $3 d_{3 / 2}$ could be deconvoluted into peaks at 367.4 , 

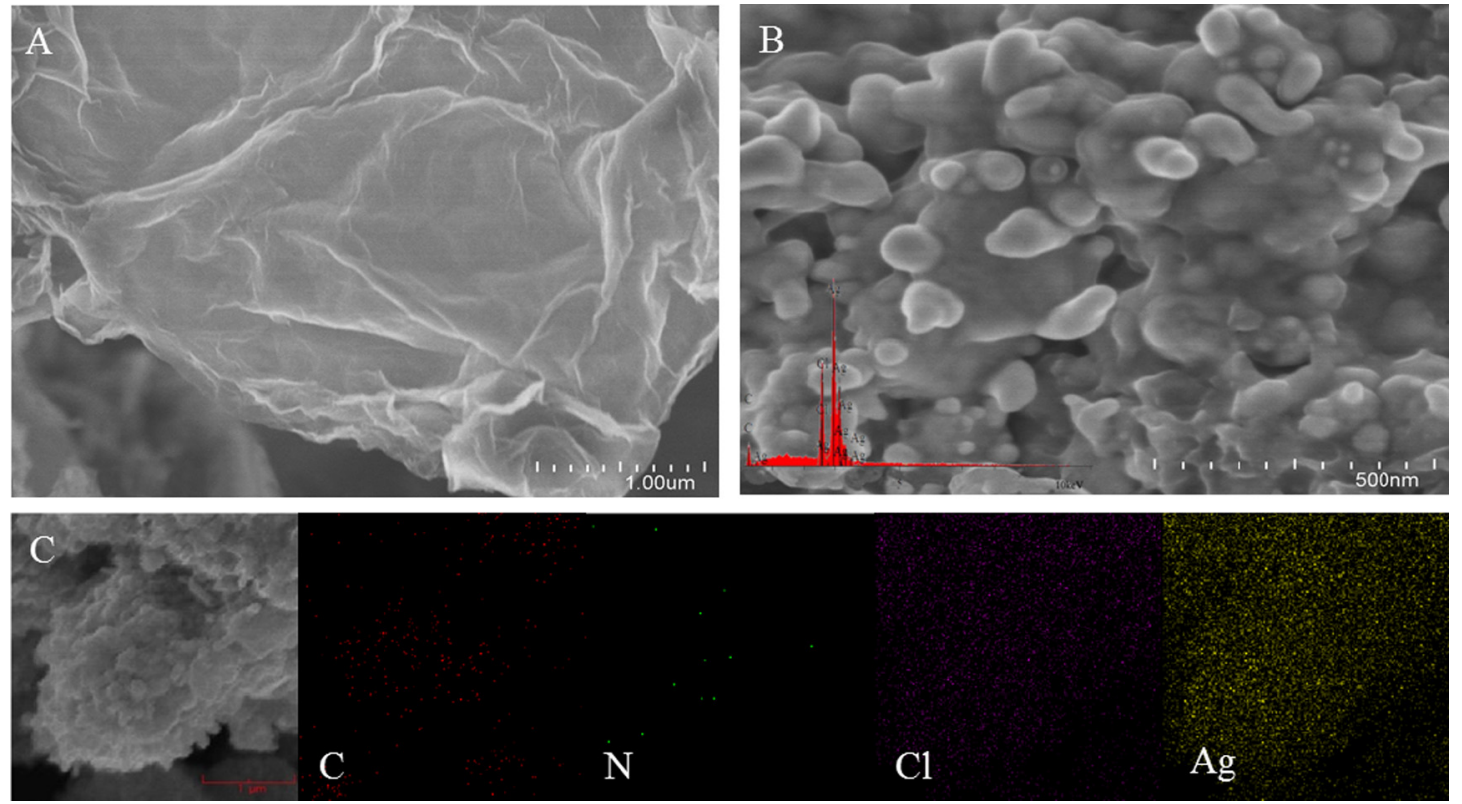

Fig. 1. FESEM images of: (A) N-rGO, (B) AgCl@Ag/N-rGO. (The inset shows the EDS spectrum of AgCl@Ag/N-rGO) and (C) the elemental mappings of AgCl@Ag/N-rGO.
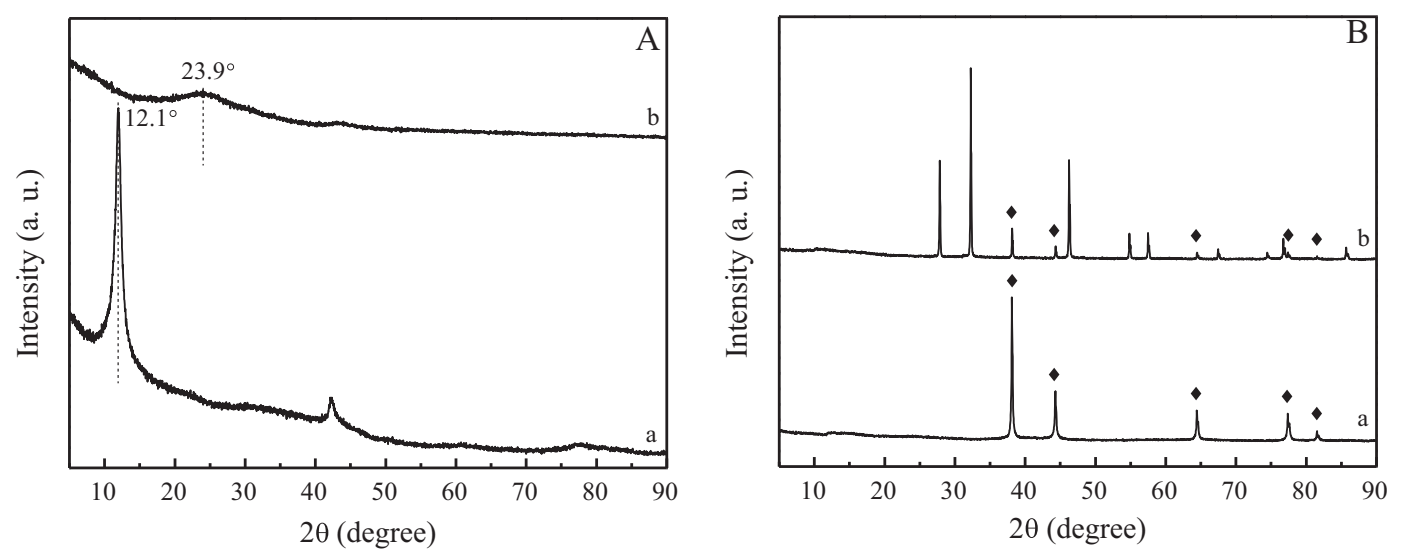

Fig. 2. XRD patterns of (A): (a) GO and (b) N-rGO. (B): (a) Ag/N-rGO and (b) AgCl@Ag/N-rGO. (•) Ag.

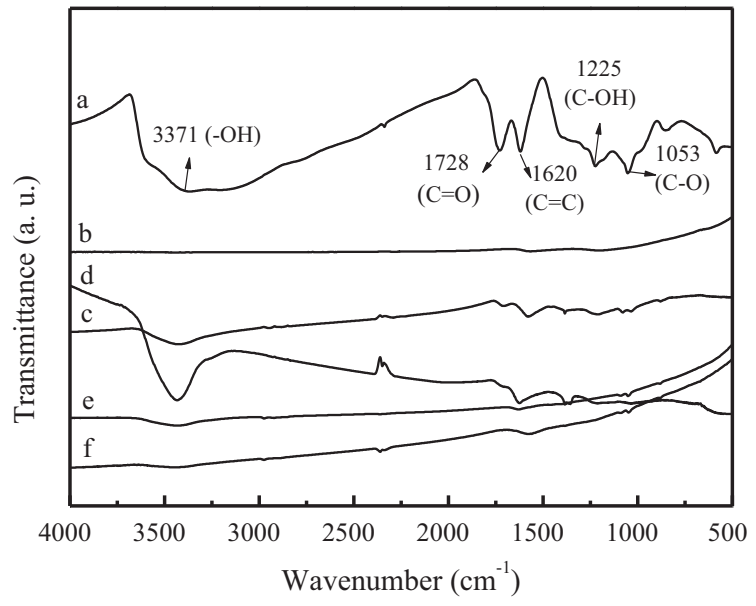

Fig. 3. FTIR spectra of (a) GO, (b) N-rGO, (c) Ag/rGO, (d) AgCl@Ag/rGO, (e) Ag/N-rGO and (f) $\mathrm{AgCl} @ \mathrm{Ag} / \mathrm{N}-\mathrm{rGO}$.

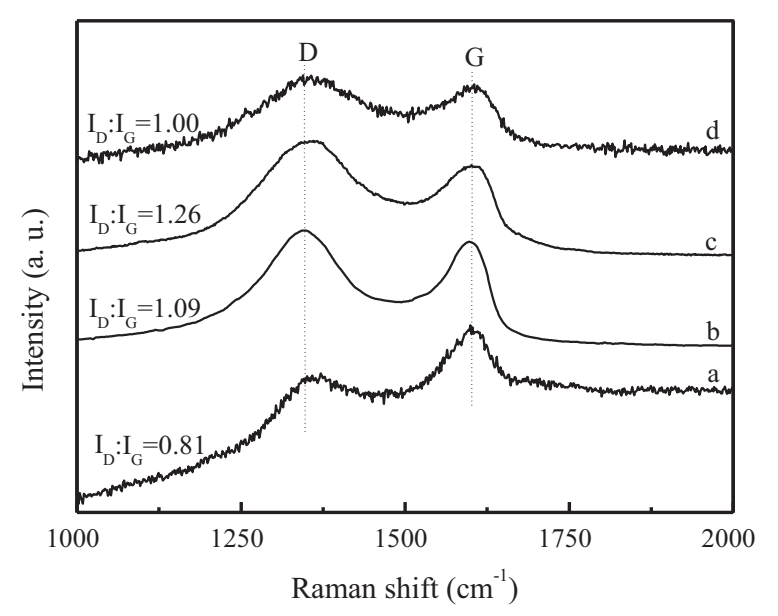

Fig. 4. Raman spectra of (a) GO, (b) N-rGO, (c) Ag/N-rGO and (d) AgCl@Ag/N-rGO. 


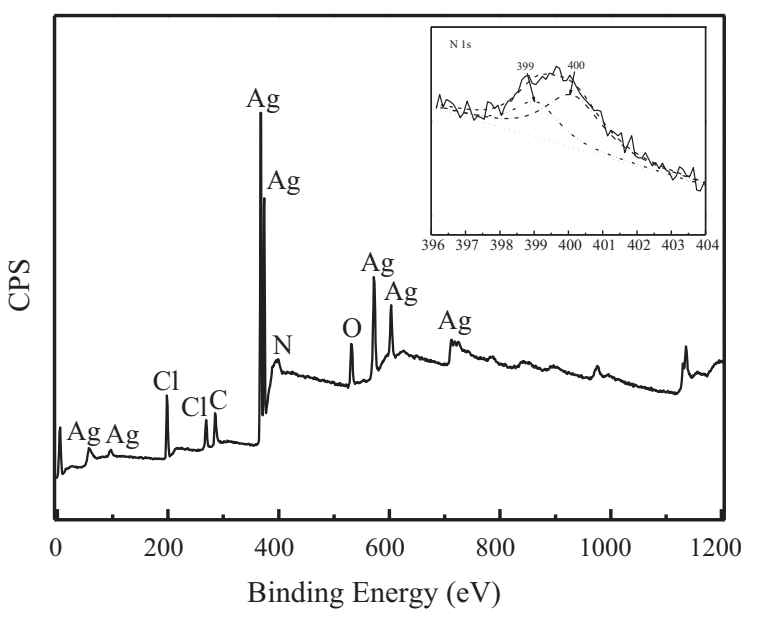

Fig. 5. An XPS spectrum of the AgCl@Ag/N-rGO photocatalyst. The inset shows a high-resolution $\mathrm{N}$ 1s spectrum with the peak deconvoluted into the pyridinic and pyrrolic $\mathrm{N}$ peaks.

$368.1 \mathrm{eV}, 373.4$ and $374.1 \mathrm{eV}$, respectively. The peaks at 367.4 and $373.4 \mathrm{eV}$ were for the $\mathrm{Ag}^{+}$of $\mathrm{AgCl}$, whereas the peaks at 368.1 and $374.1 \mathrm{eV}$ were attributed to metallic $\mathrm{Ag}^{0}$ [38]. As shown in Fig. S7B, on the basis of the calculation of the auger parameter $(=\mathrm{BE}$ $\left(\right.$ Ag3d $\left._{5 / 2}\right)$ - Auger (M4VV) 1486.71 (characteristic energy, eV)), the auger parameters of $\mathrm{AgCl} @ \mathrm{Ag} / \mathrm{N}-\mathrm{rGO}$ and $\mathrm{Ag} / \mathrm{N}-\mathrm{rGO}$ were $723.6 \mathrm{eV}$ and $725.0 \mathrm{eV}$, respectively, which could be assumed to be $\mathrm{Ag}^{+}$and $\mathrm{Ag}^{0}$ [39]. Moreover, a little shoulder at approximately $725 \mathrm{eV}$ in Ag MVV-Auger (Fig. S7B, spectrum a) indicates the presence of $\mathrm{Ag}^{0}$ [40]. The XPS results indicate that the $\mathrm{Ag}^{0}$ NPs were mainly surrounded by $\mathrm{AgCl}$.

Fig. 6 show the typical UV-visible spectra of GO, Ag/N-rGO and $\mathrm{AgCl} @ \mathrm{Ag} / \mathrm{N}-\mathrm{rGO}$. GO displayed a broad absorption band maximum between $270 \mathrm{~nm}$ and $390 \mathrm{~nm}$, which was accompanied by a tail absorption between 400 and $800 \mathrm{~nm}$. In contrast, $\mathrm{Ag} / \mathrm{N}-\mathrm{rGO}$ exhibited a strong absorption at $320 \mathrm{~nm}$, which is likely due to the electrons directly bonded to the silver atoms that acted as bulk silver [41]. Compared with GO and Ag/N-rGO, AgCl@Ag/N-rGO exhibited the strongest absorption in the visible region $(\lambda>400 \mathrm{~nm})$, and a broad absorption peak could be seen at approximately $500 \mathrm{~nm}$, which was caused by the surface plasmon resonance (SPR) of the residual $\mathrm{Ag}$ NPs after the oxidation of $\mathrm{FeCl}_{3}$.

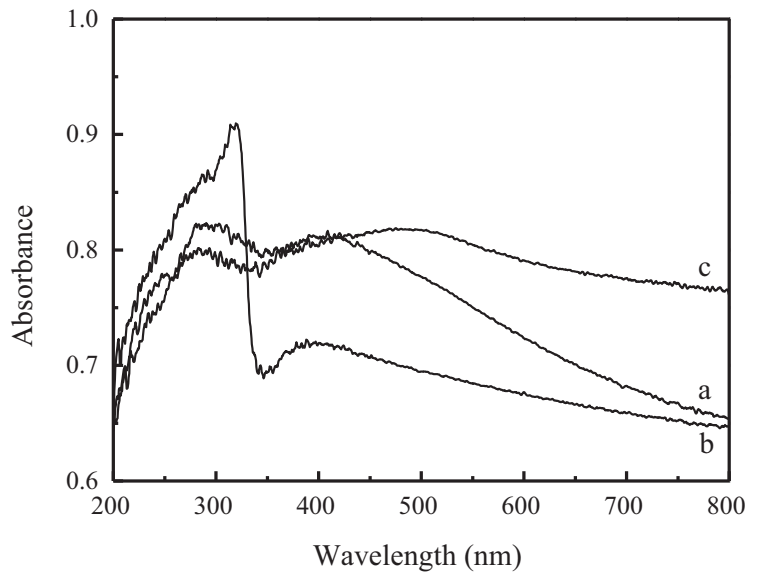

Fig. 6. UV-vis diffuse reflectance spectra of (a) GO, (b) Ag/N-rGO and (c) AgCl@Ag/ N-rGO.

\subsection{Photocatalytic performance}

The photocatalytic activity of different catalysts was evaluated by the photodegradation of 2-CP under visible light irradiation $(\lambda \geq 400 \mathrm{~nm})$ in Fig. 7. $\mathrm{Ag} / \mathrm{N}-\mathrm{rGO}, \mathrm{s}-\mathrm{Ag} / \mathrm{N}-\mathrm{rGO}$ and $\mathrm{AgCl} @ \mathrm{Ag} / \mathrm{rGO}$ could only degrade approximately $16 \%, 25 \%$ and $48 \%$ of 2 -CP within 150 min, respectively. In contrast, the AgCl@Ag/N-rGO photocatalyst exhibited the highest photocatalytic activity, and degraded approximately $97 \%$ of 2 -CP under identical experimental conditions. Correspondingly, the TOC removal of $\mathrm{AgCl} @ \mathrm{Ag} / \mathrm{rGO}$ was only $1.9 \%$; however, approximately $75.6 \%$ of the TOC was removed in the AgCl@Ag/N-rGO suspension, which was due to pyridinic N serving as highly active sites and increasing the activity of the catalyst [22]. Remarkably, the superior photocatalytic activity of $\mathrm{AgCl} @ \mathrm{Ag} / \mathrm{N}-\mathrm{rGO}$ outperformed that of $\mathrm{N}$-doped $\mathrm{TiO}_{2}$ (56.1\% of 2CP degradation and $24.7 \%$ of TOC removal), which is considered the standard example of the visible-light photocatalyst [42]. The photocatalytic activities of the related photocatalysts in previous literature, such as Ag@AgCl/RGO [24] and NG-AgCl@Ag [25], were also tested under the same conditions (Fig. S8). AgCl@Ag/N-rGO exhibited a 2-CP substrate degradation activity similar to those of $\mathrm{Ag} @ \mathrm{AgCl} / \mathrm{RGO}$ and NG-AgCl@Ag. The TOC removal of $\mathrm{Ag} @ \mathrm{AgCl} /$ RGO and NG-AgCl@Ag was 67.8\% and 72.1\%, respectively, lower than that of AgCl@Ag/N-rGO (75.6\%). Moreover, during the 2-CP degradation, the $\mathrm{Ag}^{+}$leaching concentration of $\mathrm{AgCl} @ \mathrm{Ag} / \mathrm{N}-\mathrm{rGO}$ was only $0.22 \mathrm{mg} \mathrm{L}^{-1}$, which is relatively low compared to those of $\mathrm{Ag} @ \mathrm{AgCl} / \mathrm{RGO}\left(0.34 \mathrm{mg} \mathrm{L}^{-1}\right)$ and NG-AgCl@Ag (0.49 $\left.\mathrm{mg} \mathrm{L}^{-1}\right)$ (Table S1). In addition, after five 2-CP photodegradation cycling runs, the photoactivity of $\mathrm{AgCl} @ \mathrm{Ag} / \mathrm{N}-\mathrm{rGO}$ demonstrated no significant loss, as shown in Fig. 8. The XRD patterns of AgCl@Ag/N-rGO were also unchanged after the photoreaction (Fig. S9). The results suggest that $\mathrm{AgCl} @ \mathrm{Ag} / \mathrm{N}-\mathrm{rGO}$ was an effective and stable visiblelight-driven photocatalyst due to the enhanced electron transfer resulting from the close interfacial contact between the $\mathrm{Ag}$ and $\mathrm{N}-\mathrm{rGO}$ and the in situ formation of $\mathrm{AgCl}$ around $\mathrm{Ag}$.

Industrial effluents may be basic or acidic, and thus, the effects of the initial solution $\mathrm{pH}$ on the photodegradation efficiency of 2$\mathrm{CP}$ were studied (Fig. S10). At the natural $\mathrm{pH}$ of $2-\mathrm{CP}(\mathrm{pH}=6.0)$, $\mathrm{AgCl} @ \mathrm{Ag} / \mathrm{N}-\mathrm{rGO}$ exhibited the highest photocatalytic activity. When adjusting the pH to 4.0 and 8.0 using dilute $\mathrm{HNO}_{3}$ and $\mathrm{NaOH}$, the photocatalytic activity of $2-\mathrm{CP}$ decreased. The $\mathrm{pH}$ value of the 2 -CP solution influenced the photocatalytic process for many reasons, including the surface charge state of $\mathrm{AgCl} @ \mathrm{Ag} / \mathrm{N}-\mathrm{rGO}$ and the dissociation of 2-CP. When the $\mathrm{pH}$ decreased, the active hydro-

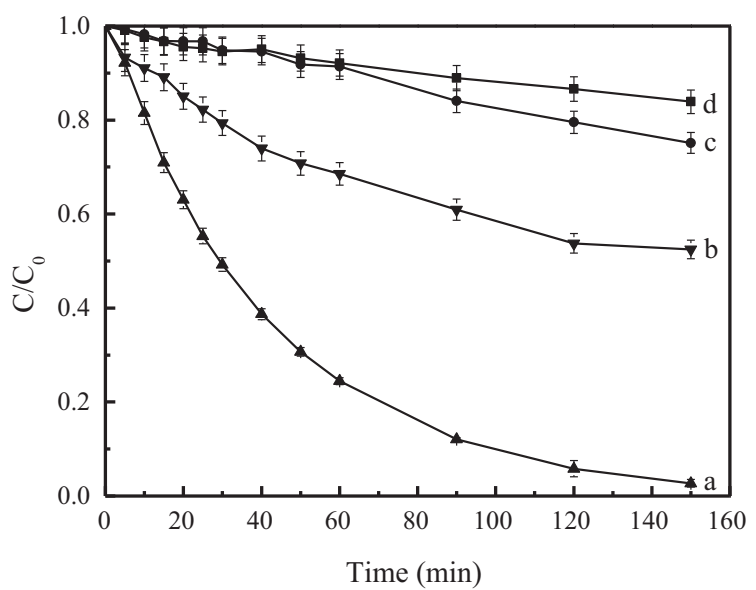

Fig. 7. The degradation of $2-\mathrm{CP}\left(10 \mathrm{mg} \mathrm{L}^{-1}\right)$ in different photocatalyst suspensions (1.6 $\left.\mathrm{g} \mathrm{L}^{-1}\right)$ under visible light $(\lambda>400 \mathrm{~nm})$ : (a) AgCl@Ag/N-rGO, (b) AgCl@Ag/rGO, (c) $\mathrm{s}-\mathrm{Ag} / \mathrm{N}-\mathrm{rGO}$ and (d) $\mathrm{Ag} / \mathrm{N}-\mathrm{rGO}$. (The data were the average of the triplicates with a standard deviation of less than $5 \%$ ). 


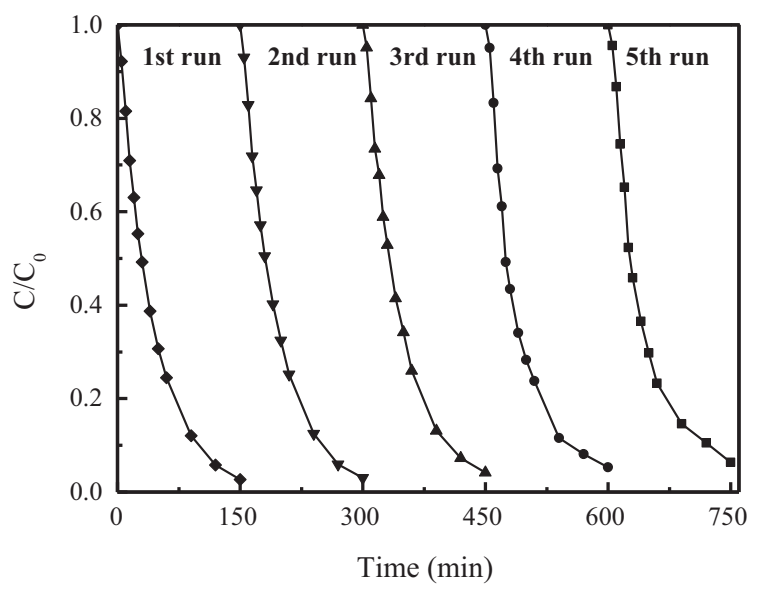

Fig. 8. Cycling runs in the photodegradation of 2-CP $\left(10 \mathrm{mg} \mathrm{L}^{-1}\right)$ in an $\mathrm{AgCl} @ \mathrm{Ag} / \mathrm{N}-$ rGO aqueous dispersion (1.6 $\left.\mathrm{g} \mathrm{L}^{-1}\right)$ under visible light $(\lambda \geq 400 \mathrm{~nm})$.

xyl groups on the catalyst surface decreased accordingly, resulting in a decrease in the generated ${ }^{\circ} \mathrm{OH}$ and causing slowed reaction rate for the 2-CP degradation [43]. When the $\mathrm{pH}$ increased to 8.0, 2-CP $(\mathrm{pKa}=8.56)$ was probably dissociated into chlorophenoxide ions, which would compete for the reactive sites with the hydroxyl groups and reduce the $\mathrm{OH}$ radicals. Meanwhile, the repulsion between the negatively charged surface of $\mathrm{AgCl} @ \mathrm{Ag} / \mathrm{N}-\mathrm{rGO}$ (the $\mathrm{pH}$ of the point of zero charge, $\mathrm{pH}_{\mathrm{pzc}}=1.70$, Fig. S10) and chlorophenoxide ions at high $\mathrm{pH}$ could also decrease the photocatalytic degradation rate of 2-CP.

In addition, three other aromatic pollutants (i.e., BPA, phenol and $2,4-\mathrm{D}$ ) were degraded more than $82 \%, 82 \%$ and $60 \%$, respectively, at $150 \mathrm{~min}$ and the corresponding TOC removals were $52.3 \%, 51.8 \%$ and $25.9 \%$ (Fig. 9). However, only $25 \%$ of DP with Ncontaining side-chains could be removed, and the TOC removal was only $4.9 \%$. More specially, PHT with $\mathrm{N}$-containing heterocyclic ring could not be degraded by AgCl@Ag/N-rGO after a 150-min reaction under visible light. In fact, the $\mathrm{N}$ functional groups in $\mathrm{N}-$ rGO are Lewis-base sites [44] that repel the pollutants with $\mathrm{N}$ containing side-chains or heterocyclic rings in which the $\mathrm{N}$ heteroatoms can serve as Lewis bases [45]. Therefore, the photoactivity of $\mathrm{AgCl} @ \mathrm{Ag} / \mathrm{N}-\mathrm{rGO}$ was related to the structure of the organic substances, and the interaction between $\mathrm{N}-\mathrm{rGO}$ and pollutants was probably partly responsible for the removal of pollutants.

\subsection{Photocatalytic mechanism}

To investigate the active species in the photocatalytic process of $\mathrm{AgCl} @ \mathrm{Ag} / \mathrm{N}-\mathrm{rGO}$ under visible light irradiation, the effects of various radical scavengers on the degradation of 2-CP were shown in Fig. 10. The degradation of 2-CP was markedly depressed by $\mathrm{p}-$ benzoquinone ( $\mathrm{p}-\mathrm{BQ}, 1 \mathrm{mM})$, indicating that the superoxide anion radicals $\left(\mathrm{O}_{2}{ }^{--}\right)$were the main reactive oxygen species in the reaction. In the presence of sodium oxalate $\left(\mathrm{Na}_{2} \mathrm{C}_{2} \mathrm{O}_{4}, 5 \mathrm{mM}\right)$, the rate decreased by $30 \%$, illustrating that the photogenerated holes $\left(\mathrm{h}^{+}\right)$ were partly responsible for the removal of 2-CP. In addition, the degradation of 2-CP was depressed to some extent with the addition of $\mathrm{NaHCO}_{3}(100 \mathrm{mM})$, but little change was noted with the addition of tert-butanol (TBA, 100 mM) in the AgCl@Ag/N-rGO suspension, suggesting the existence of surface-adsorbed $\mathrm{OH}$ in the reaction [42].

The ESR technique with BMPO was employed to further explore the reactive species. As shown in Fig. 11A, the characteristic peaks of BMPO-OH in AgCl@Ag/N-rGO were obviously visible. However, no such signals were detected in the $\mathrm{Ag} / \mathrm{N}-\mathrm{rGO}$ suspension under visible light $(\lambda \geq 400 \mathrm{~nm})$ irradiation. The result was consistent with the active species trapping experiment. In Fig. 11B, the similar

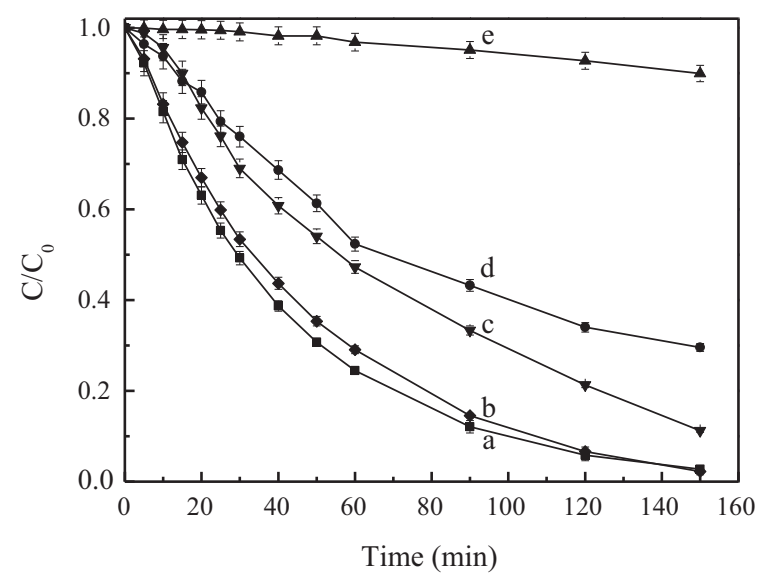

Fig. 10. Plotted degradation kinetics of 2-CP $\left(10 \mathrm{mg} \mathrm{L}^{-1}\right)$ in the $\mathrm{AgCl} @ \mathrm{Ag} / \mathrm{N}-\mathrm{rGO}$ suspension (1.6 $\left.\mathrm{g} \mathrm{L}^{-1}\right)$ under visible light $(\lambda \geq 400 \mathrm{~nm}$ ) with (a) no scavenger added, (b) TBA (100 mM), (c) $\mathrm{NaHCO}_{3}(100 \mathrm{mM})$, (d) $\mathrm{Na}_{2} \mathrm{C}_{2} \mathrm{O}_{4}(5 \mathrm{mM})$ and (e) p-BQ (1 mM). (The data were the average of the triplicates with a standard deviation of less than $5 \%)$.
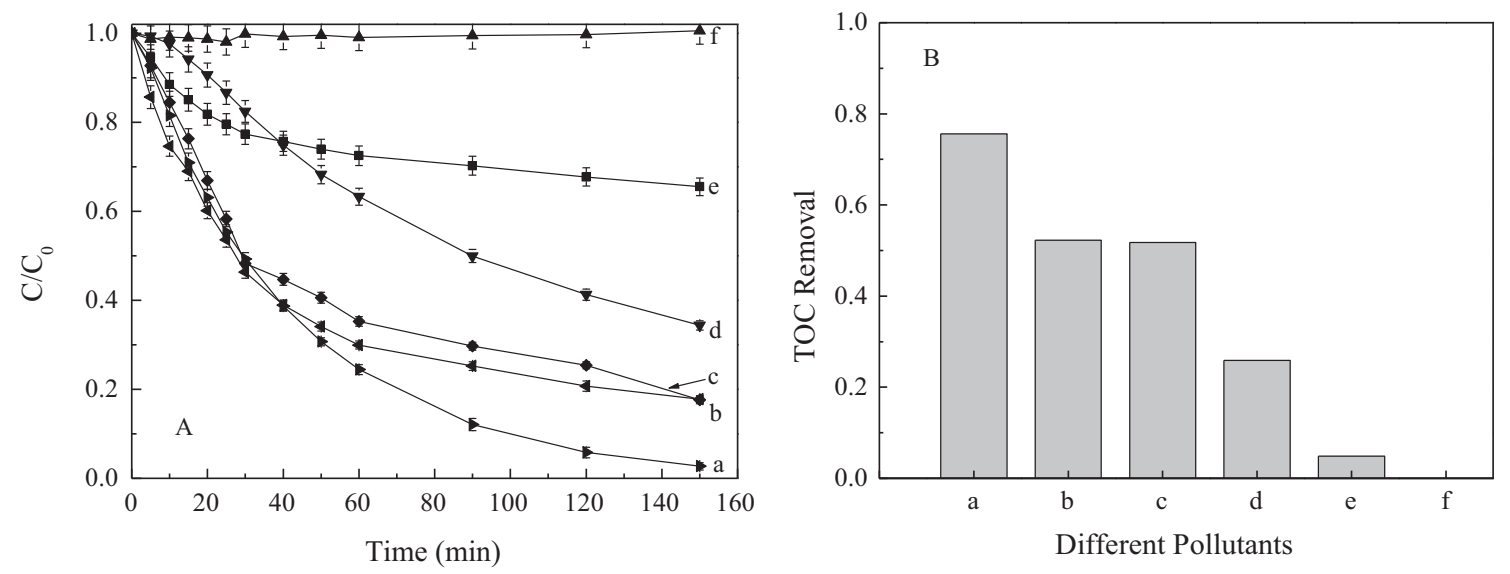

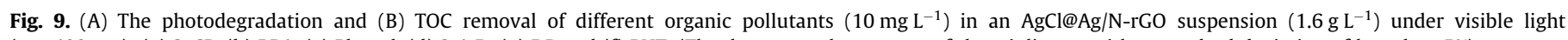
$(\lambda \geq 400 \mathrm{~nm})$ : (a) 2-CP, (b) BPA, (c) Phenol, (d) 2,4-D, (e) DP and (f) PHT. (The data were the average of the triplicates with a standard deviation of less than 5\%). 

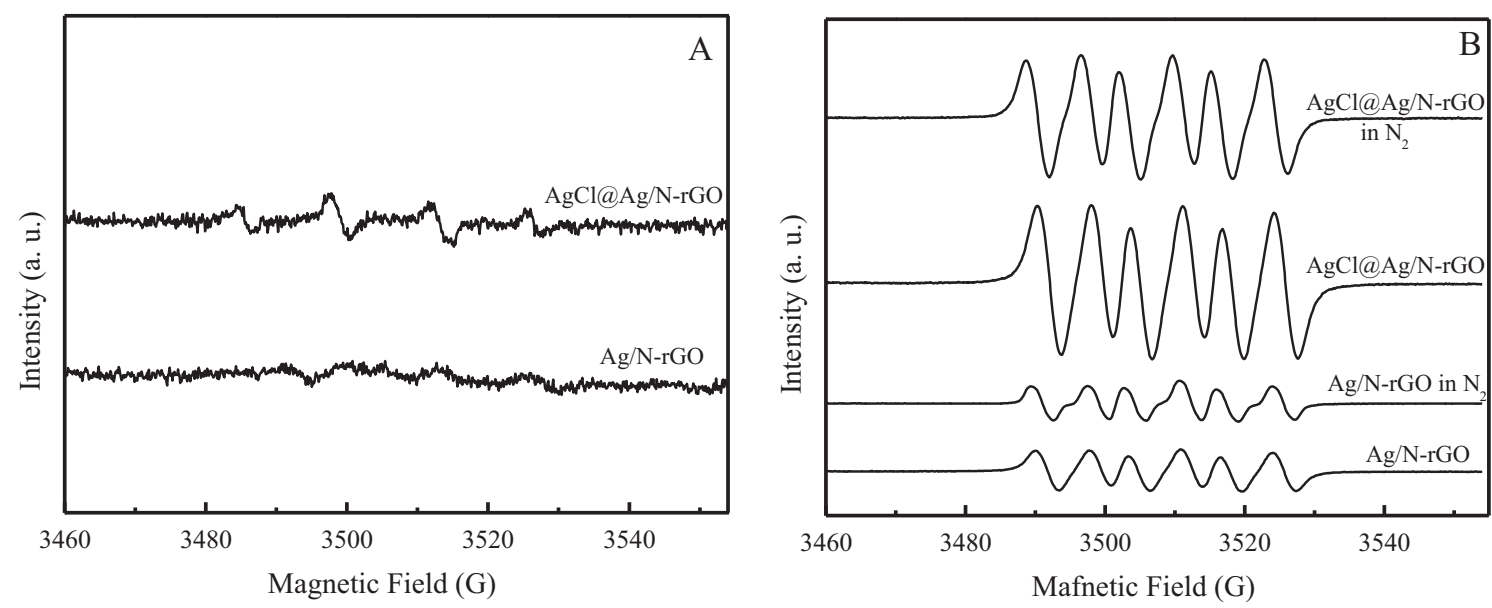

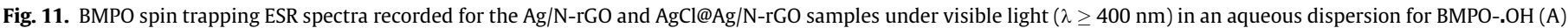
and in a methanol dispersion for $\mathrm{BMPO}^{-\mathrm{O}_{2} \cdot-}$ (B).

signals of BMPO- $\mathrm{O}_{2}^{--}$were observed under air and $\mathrm{N}_{2}$-saturated conditions, indicating that the photogenerated electrons could reduce the adsorbed $\mathrm{O}_{2}$ on the catalyst surface to form $\mathrm{O}_{2}{ }^{-}$. Compared with $\mathrm{Ag} / \mathrm{N}-\mathrm{rGO}, \mathrm{AgCl} @ \mathrm{Ag} / \mathrm{N}-\mathrm{rGO}$ could produce more $\mathrm{O}_{2}{ }^{-}$, indicating that the SPR of the $\mathrm{Ag}$ NPs and the $\mathrm{AgCl}$ at the surface could promote the electron transfer to the catalyst surface, resulting in the generation of more $\mathrm{O}_{2}{ }^{--}$.

To illustrate the charge-transfer processes, cyclic voltammetry analyses were performed under air-saturated and $\mathrm{N}_{2}$-saturated conditions, shown in Fig. 12. No peaks appeared for the $\mathrm{Ag} / \mathrm{N}$ rGO catalyst before and after light irradiation under $\mathrm{N}_{2}$-saturated
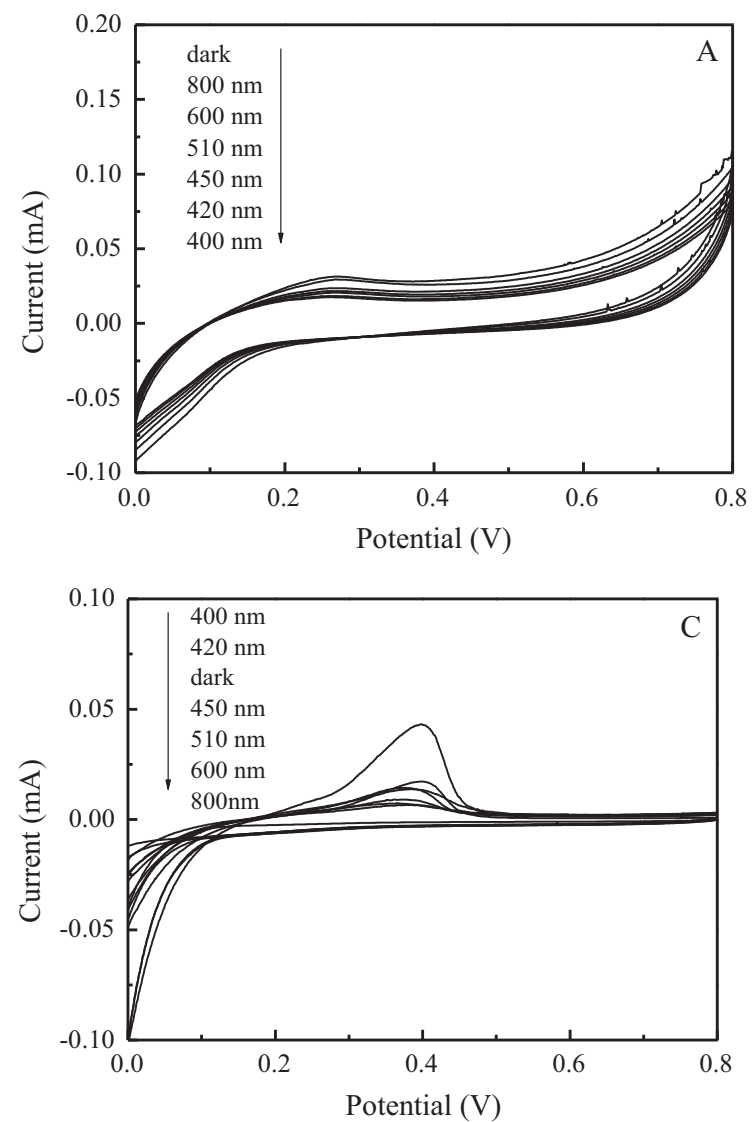

condition (Fig. 12A). The Ag/N-rGO sample exhibited a pair of well-defined redox peaks corresponding to the oxidation and reduction of $\mathrm{Ag}$ in dark and air-saturated conditions (Fig. 12B), but the peaks disappeared under light irradiation with the tested wavelengths, indicating that Ag could be oxidized by oxygen in the solution in the dark. Meanwhile, N-rGO as an electron donor could transfer electrons to $\mathrm{Ag}$ after light irradiation. Under $\mathrm{N}_{2}$ saturated conditions, a weak oxidation peak for $\mathrm{Ag}$ was detected at the AgCl@Ag/N-rGO photoanode in the dark (Fig. 12C). When the $\mathrm{AgCl} @ \mathrm{Ag} / \mathrm{N}-\mathrm{rGO}$ was irradiated by light with the wavelengths $\lambda \geq 800$ and $\lambda \geq 600 \mathrm{~nm}$, no oxidation peak for $\mathrm{Ag}$ appeared, fur-
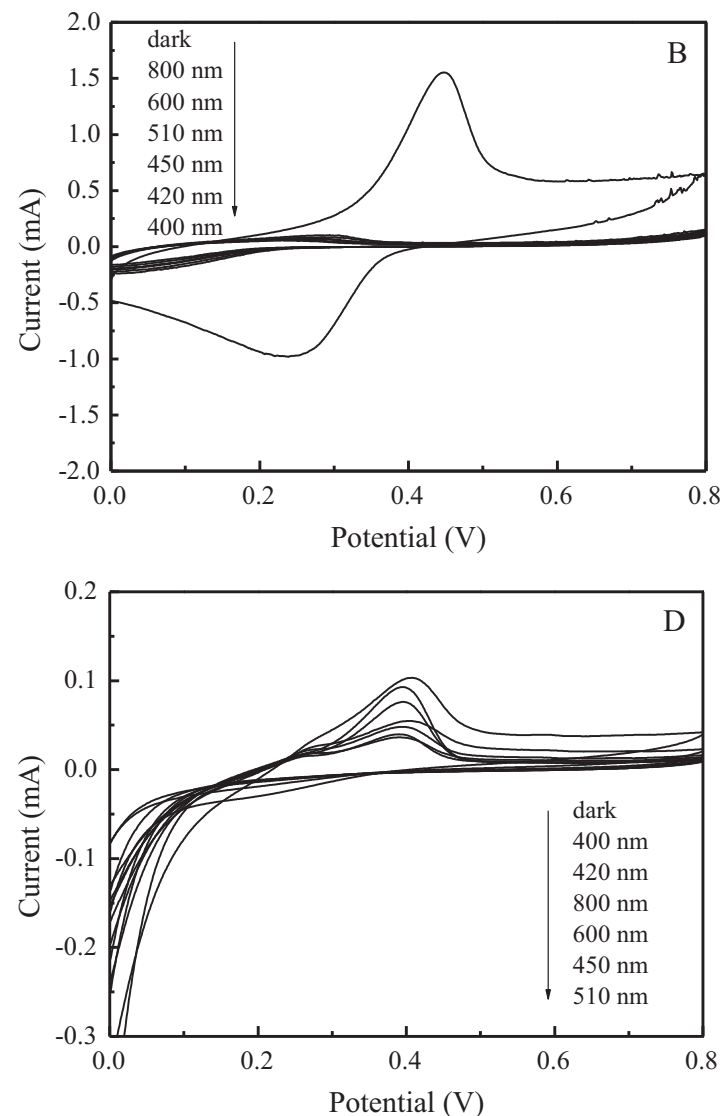

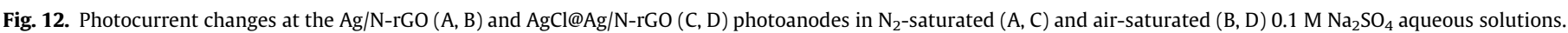




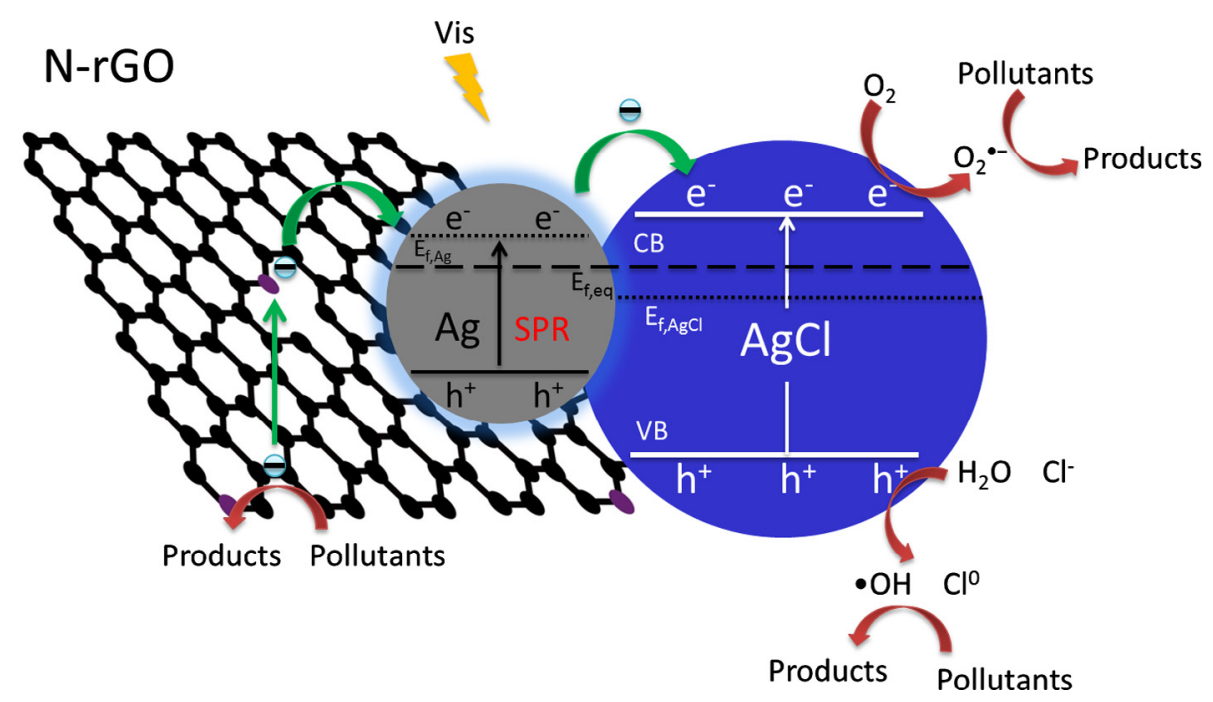

Fig. 13. The photocatalytic mechanism for AgCl@Ag/N-rGO composite photocatalyst.

ther indicating that $\mathrm{N}-\mathrm{rGO}$ could transfer electrons to Ag after light irradiation. Furthermore, a weak peak could be seen under light irradiation with the wavelengths $\lambda \geq 510$ to $\lambda \geq 400 \mathrm{~nm}$, which could be assigned to the plasmon-induced charge separation of Ag NPs. A similar phenomenon was observed in air at the AgCl@Ag/N-rGO photoanode (Fig. 12D). In contrast, the oxidation peak of Ag NPs was observed even in the dark due to the presence of oxygen and showed the strongest intensity. Under visible-light irradiation, the electrons from the plasmon-induced Ag NPs transferred to the conduction band of $\mathrm{AgCl}$ and were then trapped by oxygen to form $\mathrm{O}_{2}{ }^{-}$. Meanwhile, electrons from $\mathrm{N}-\mathrm{rGO}$ could be transferred to the Ag NPs to reduce the oxidized silver. Therefore, the oxidation peak of Ag NPs became weaker after light irradiation than before in air.

Herein, based on the above results, the photocatalysis mechanism was illustrated in Fig. 13. Under visible light irradiation, photogenerated electron-hole pairs could be formed in the Ag NPs due to the SPR. Meanwhile, some electron-hole pairs existed in the $\mathrm{AgCl}$ due to the self-photosensitivity [10]. The increased electron density caused the Fermi level disequilibrium. Thus, the electrons were transferred into the conduction band of the $\mathrm{AgCl}$ until the Fermi level of the system achieved equilibrium, resulting in the formation of $\mathrm{O}_{2}{ }^{--}$on the surface of the $\mathrm{AgCl}$ [46]. Based on the cyclic voltammetry analyses, the other electron transfer occurred from $\mathrm{N}$-rGO to the Ag NPs, which could decrease the $\mathrm{Ag}^{+}$leaching to keep the system stable. Meanwhile, pollutants could lose electrons to $\mathrm{N}-\mathrm{rGO}$ by the interaction between pollutants and $\mathrm{N}-\mathrm{rGO}$, and then were oxidized. On the other hand, the holes on the valence band of $\mathrm{AgCl}$ could oxidize $\mathrm{H}_{2} \mathrm{O}$ to form $\mathrm{OH}$ [47], which was also confirmed by the ESR result. Moreover, the generated holes at the valence band of the $\mathrm{AgCl}$ could oxidize the $\mathrm{Cl}^{-}$ions to $\mathrm{Cl}^{0}$ atoms [48], which could also degrade and mineralize the organic pollutants. Therefore, the high photoactivity and photostability of AgCl@Ag/N-rGO could be obtained by the fast charge separation and transfer to form $\mathrm{O}_{2}{ }^{-}$, excited $\mathrm{h}^{+}$, and ${ }^{\circ} \mathrm{OH}$ and $\mathrm{Cl}^{0}$ radicals on the catalyst surface for removing pollutants.

\section{Conclusions}

A plasmonic photocatalyst AgCl@Ag/N-rGO with close interfacial contact was synthesized by a hydrothermal-in situ oxidation method. The AgCl@Ag/N-rGO photocatalyst was highly efficient and stable for the degradation and mineralization of various pollu- tants under visible light, such as 2-CP, BPA, phenol and 2,4-D. Compared to $\mathrm{AgCl} @ \mathrm{Ag} / \mathrm{rGO}$ and other related photocatalysts, $\mathrm{AgCl} @ \mathrm{Ag} /$ $\mathrm{N}-\mathrm{rGO}$ demonstrated optimal performance properties. The strengthened photocatalytic performance could be attributed to the SPR effects of the Ag NPs and the close interfacial contact in AgCl@Ag/N-rGO, promoting the separation and migration of photogenerated electrons and holes. Two electron transfer processes were verified from $\mathrm{Ag}$ to $\mathrm{AgCl}$ and from $\mathrm{N}-\mathrm{rGO}$ to $\mathrm{Ag}$, and pollutants could lose electrons to N-rGO through this interaction. Additionally, the active species $\left(\mathrm{O}_{2}^{-{ }^{-}}, \mathrm{h}^{+}\right.$and surface-adsorbed $\left.\cdot \mathrm{OH}\right)$ were formed to degrade organic pollutants in the photoreaction system of AgCl@Ag/N-rGO. This study provides a new perspective in the development of visible-light-driven photocatalysts for water pollution purification and solar energy conversion.

\section{Acknowledgment}

This work was supported by National Key Research and Development Plan [2016YFA0203204] and the National Natural Science Foundation of China [Grant Numbers 51538013, 21407165, 51278527, 51638011].

\section{Appendix A. Supplementary material}

Supplementary data associated with this article can be found, in the online version, at http://dx.doi.org/10.1016/j.jcis.2017.06.037.

\section{References}

[1] Z. Zou, J. Ye, K. Sayama, H. Arakawa, Direct splitting of water under visible light irradiation with an oxide semiconductor photocatalyst, Nature 414 (2001) 625-627.

[2] Y. Peng, K.K. Wang, T. Liu, J. Xu, B.G. Xu, Synthesis of one-dimensional $\mathrm{Bi}_{2} \mathrm{O}_{3}-$ $\mathrm{Bi}_{2} \mathrm{O}_{2.33}$ heterojunctions with high interface quality for enhanced visible light photocatalysis in degradation of high-concentration phenol and MO dyes, Appl. Catal. B: Environ. 203 (2017) 946-954

[3] C.N. Tang, E.Z. Liu, J. Wan, X.Y. Hu, J. Fan, $\mathrm{CO}_{3} \mathrm{O}_{4}$ nanoparticles decorated $\mathrm{Ag}_{3} \mathrm{PO}_{4}$ tetrapods as an efficient visible-light-driven heterojunction photocatalyst, Appl. Catal. B: Environ. 181 (2016) 707-715.

[4] Y.Q. Yang, G. Liu, J.T.S. Irvine, H.M. Cheng, Enhanced photocatalytic $\mathrm{H}_{2}$ production in core-shell engineered rutile $\mathrm{TiO}_{2}$, Adv. Mater. 28 (2016) 58505856.

[5] J.L. Li, X.J. Liu, Z. Sun, Y. Sun, L.K. Pan, Novel yolk-shell structure bismuth-rich bismuth molybdate microspheres for enhanced visible light photocatalysis, J. Colloid Interf. Sci. 452 (2015) 105-119. 
[6] Q. Tian, X.J. Yu, L.F. Zhang, D.M. Yu, Monodisperse raspberry-like multihollow polymer/Ag nanocomposite microspheres for rapid catalytic degradation of methylene blue, J. Colloid Interf. Sci. 491 (2017) 294-304.

[7] R. Asapu, N. Claes, S. Bals, S. Denys, C. Detavernier, S. Lenaerts, S.W. Verbruggen, Silver-polymer core-shell nanoparticles for ultrastable plasmonenhanced photocatalysis, Appl. Catal. B: Environ. 200 (2017) 31-38.

[8] C. An, S. Wang, Y. Sun, Q. Zhang, J. Zhang, C. Wang, J. Fang, Plasmonic silver incorporated silver halides for efficient photocatalysis, J. Mater. Chem. A 4 (2016) 4336-4352.

[9] W. Wu, X.M. Lv, J.X. Wang, J.M. Xie, Integrating AgI/AgBr biphasic heterostructures encased by few layer $h$-BN with enhanced catalytic activity and stability, J. Colloid Interf. Sci. 496 (2017) 434-445.

[10] Z.Y. Lin, J. Xiao, J.H. Yan, P. Liu, L.H. Li, G.W. Yang, Ag/AgCl plasmonic cubes with ultrahigh activity as advanced visible-light photocatalysts for photodegrading dyes, J. Mater. Chem. A 3 (2015) 7649-7658.

[11] X.L. Xiao, L. Ge, C.C. Han, Y.J. Li, Z. Zhao, Y.J. Xin, S.M. Fang, L.N. Wu, P. Qiu, A facile way to synthesize Ag@AgBr cubic cages with efficient visible-lightinduced photocatalytic activity, Appl. Catal. B: Environ. 163 (2015) 564-572.

[12] B. Zhang, X. Wang, Y.L. Zhao, J. Lv, H.Y. Meng, H.H. Chang, H. Zhang, W.L. Wei, Highly photosensitive colorimetric immunoassay for tumor marker detection based on $\mathrm{Cu}^{2+}$ doped Ag-AgI nanocomposite, Talanta 167 (2017) 111-117.

[13] S.T. Gao, T. Feng, C. Feng, N.Z. Shang, C. Wang, Novel visible-light-responsive Ag/AgCl@MIL-101 hybrid materials with synergistic photocatalytic activity, J. Colloid Interf. Sci. 466 (2016) 284-290.

[14] X. Lin, J.G. Yu, S. Wageh, A.A. Al-Ghamdi, J. Xie, Graphene in photocatalysis: a review, Small 12 (2016) 6640-6696.

[15] T.L. Wu, C.H. Ye, W.T. Hsiao, P.Y. Huang, M.J. Huang, Y.H. Chiang, C.H. Cheng, R. S. Liu, P.W. Chiu, High-Performance organic light-emitting diode with substitutionally boron-doped graphene Anode, ACS Appl. Mater. Interf. 9 (2017) 14998-15004.

[16] D. Liu, L.B. Li, T.Y. You, Superior catalytic performances of platinum nanoparticles loaded nitrogen-doped graphene toward methanol oxidation and hydrogen evolution reaction, J. Colloid Interf. Sci. 487 (2017) 330-335.

[17] A. Kumar, K. Banerjee, M. Dvorak, F. Schulz, A. Harju, P. Rinke, P. Liljeroth, Charge-Transfer-Driven nonplanar adsorption of F4TCNQ molecules on epitaxial graphene, ACS Nano. 10.1021/acsnano.7b01599.

[18] H.L. Guo, P. Su, X. Kang, S.K. Ning, Synthesis and characterization of nitrogendoped graphene hydrogels by hydrothermal route with urea as reducingdoping agents, J. Mater. Chem. A 1 (2013) 2248-2255.

[19] H.B. Wang, T. Maiyalagan, X. Wang, Review on recent progress in nitrogendoped graphene: synthesis, characterization, and its potential applications, ACS Catal. 2 (2012) 781-794.

[20] C.Y. Jae, K.H. Sung, I. Hyungsoon, M. Yoon, J.G. Bok, L.C. Woo, P. Jeunghee, P. MiHee, C. Jaephil, K.H. Seok, Nitrogen-doped graphitic layers deposited on silicon nanowires for efficient lithium-ion battery anodes, J. Phys. Chem. C 115 (2011) 9451-9457.

[21] Y. Liang, Y. Li, H. Wang, J. Zhou, J. Wang, T. Regier, H. Dai, $\mathrm{CO}_{3} \mathrm{O}_{4}$ nanocrystals on graphene as a synergistic catalyst for oxygen reduction reaction, Nat. Mater. 10 (2011) 780-786.

[22] A. Pendashteha, J. Palmaa, M. Andersona, R. Marcillaa, NiCoMnO nanoparticles on $\mathrm{N}$-doped graphene: Highly efficient bifunctiona electrocatalyst for oxygen reduction/evolution reactions, Appl. Catal. B: Environ. 201 (2017) 241-252.

[23] H.Y. Li, S.Y. Gan, H.Y. Wang, D.X. Han, L. Niu, Intercorrelated superhybrid of $\mathrm{AgBr}$ supported on graphitic- $\mathrm{C}_{3} \mathrm{~N}_{4}$-decorated nitrogen-noped graphene: high engineering photocatalytic activities for water purification and $\mathrm{CO}_{2}$ reduction, Adv. Mater. 27 (2015) 6906-6913.

[24] H. Zhang, X.F. Fan, X. Quan, S. Chen, H.T. Yu, Graphene sheets grafted Ag@AgC hybrid with enhanced plasmonic photocatalytic activity under visible light, Environ. Sci. Technol. 45 (2011) 5731-5736.

[25] C. Dong, K.L Wu, X.W. Wei, J. Wang, L. Liu, B.B. Jiang, Nitrogen-doped graphene modified AgX@Ag ( $\mathrm{X}=\mathrm{Br}, \mathrm{Cl})$ composites with improved visible light photocatalytic activity and stability, Appl. Catal. A: General 488 (2014) 11-18.

[26] L.L. Zhang, C. Hu, H.H. Ji, p-AgI anchored on 001 facets of n-Bi2O2CO3 sheet with enhanced photocatalytic activity and stability, Appl. Catal. B: Environ. 205 (2017) 34-41

[27] Y. Xu, H. Bai, G. Lu, C. Li, G. Shi, Flexible graphene films via the filtration of water-soluble noncovalent functionalized graphene sheets, J. Am. Chem. Soc. 130 (2008) 5856-5857.
[28] Y. Min, G. He, Q. Xu, Y. Chen, Self-assembled encapsulation of graphene oxide/ $\mathrm{Ag} @ \mathrm{AgCl}$ as a Z-scheme photocatalytic system for pollutant removal, J. Mater. Chem. A 2 (2014) 1294-1301.

[29] L.T. Soo, K.S. Loh, A.B. Mohamad, W.R.W. Daud, W.Y. Wong, Synthesis of silver/ nitrogen-doped reduced graphene oxide through a one-step thermal solidstate reaction for oxygen reduction in an alkaline medium, J. Power Sources 324 (2016) 412-420.

[30] Q. Li, B. Guo, J. Yu, J. Ran, B. Zhang, H. Yan, J. Gong, Highly efficient visible-lightdriven photocatalytic hydrogen production of CdS-cluster-decorated graphene nanosheets, J. Am. Chem. Soc. 133 (2011) 10878-10884.

[31] W.P. Ouyang, D.R. Zeng, X. Yu, F.Y. Xie, W.H. Zhang, J. Chen, J. Yan, F.J. Xie, L. Wang, H. Meng, D.S. Yuan, Exploring the active sites of nitrogen-doped graphene as catalysts for the oxygen reduction reaction, Int. J. Hydrogen Energy 39 (2014) 15996-16005.

[32] M. Tajabadi, W. Basirun, F. Lorestani, R. Zakaria, S. Baradaran, Y. Amin, M. Mahmoudian, M. Rezayi, M. Sookhakian, Nitrogen-doped graphene-silver nanodendrites for the non-enzymatic detection of hydrogen peroxide, Electrochim. Acta 151 (2015) 126-133.

[33] O. Liu, H. Zhang, H. Zhong, S. Zhang, S. Chen, N-doped graphene/carbon composite as non-precious metal electrocatalyst for oxygen reduction reaction, Electrochim. Acta 81 (2012) 313-320.

[34] C. Cui, Y. Wang, D. Liang, W. Cui, H. Hu, B. Lu, S. Xu, X. Li, C. Wang, Y. Yang, Photo-assisted synthesis of $\mathrm{Ag}_{3} \mathrm{PO}_{4} /$ reduced graphene oxide/Ag heterostructure photocatalyst with enhanced photocatalytic activity and stability under visible light, Appl. Catal. B: Environ. 158-159 (2014) 150-160.

[35] J. Chen, M. Wang B. Liu, Z Fan, K. Cui, Y. Kuang Platinum catalysts prepared with functional carbon nanotube defects and its improved catalytic performance for methanol oxidation, J. Phys. Chem. B 110 (2006) 1177511779.

[36] A.P. Periasamy, J. Liu, H. Lin, H. Chang, Synthesis of copper nanowire decorated reduced graphene oxide for electro-oxidation of methanol, J. Mater. Chem. A 1 (2013) 5973-5981

[37] H. Liu, M. Jia, B. Cao, R. Chen, X. Lv, R. Tang, F. Wu, B. Xu, Nitrogen-doped carbon/graphene hybrid anode material for sodiumion batteries with excellent rate capability, J. Power Sources 319 (2016) 195-201.

[38] B. Cai, J. Wang, S.Y. Gan, D.X. Han, Z.J. Wu, L. Niu, A distinctive red Ag/AgCl photocatalyst with efficient photocatalytic oxidative and reductive activities, J. Mater. Chem. A 2 (2014) 5280-5286.

[39] C. Hu, Y. Lan, J. Qu, X. Hu, A. Wang, $\mathrm{Ag} / \mathrm{AgBr} / \mathrm{TiO}_{2}$ visible light photocatalyst for destruction of azodyes and bacteria, J. Phys. Chem. B 110 (2006) 4066-4072.

[40] S.G. Aspromonte, M.D. Mizrahi, F.A. Schneeberger, J.M.R. López, A.V. Boix, Study of the nature and location of silver in Ag-exchanged mordenite catalysts characterization by spectroscopic techniques, J. Phys. Chem. C 117 (2013) 25433-25442.

[41] H. Hu, Z. Jiao, G. Lu, J. Ye, Y. Bi, Enhanced photocatalytic properties of biomimetic Ag/AgCl heterostructures, RSC Adv. 4 (2014) 31795-31798.

[42] C. Hu, T.W. Peng, X.X. Hu, Y.L. Nie, X.F. Zhou, J.H. Qu, H. He, Plasmon-induced photodegradation of toxic pollutants with $\mathrm{Ag}-\mathrm{AgI} / \mathrm{Al}_{2} \mathrm{O}_{3}$ under visible-light irradiation, J. Am. Chem. Soc. 132 (2010) 857-862.

[43] M. Khraisheh, L. Wu, A.H. Al-Muhtaseb, A.B. Albadarin, G.M. Walker, Phenol degradation by powdered metal ion modified titanium dioxide photocatalysts, Chem. Eng. J. 213 (2012) 125-134.

[44] X. Wang, Y. Qin, L. Zhu, H. Tang, Nitrogen-doped reduced graphene oxide as a bifunctional material for removing bisphenols: Synergistic effect between adsorption and catalysis, Environ. Sci. Technol. 49 (2015) 6855-6864.

[45] L. Wang, D. Zhu, L. Duan, W. Chen, Adsorption of single-ringed N- and Sheterocyclic aromatics on carbon nanotubes, Carbon 48 (2010) 3906-3915.

[46] M.M. Liu, L.A. Hou, B.D. Xi, Q. Li, X.J. Hu, S.L. Yu, Magnetically separable Ag/ $\mathrm{AgCl}$-zero valent iron particles modified zeolite $\mathrm{X}$ heterogeneous photocatalysts for tetracycline degradation under visible light, Chem. Eng. J. 302 (2016) 475-484.

[47] H.Y. Yin, X.L. Wang, L. Wang, Q.L. Nie, Y. Zhang, Q.L. Yuan, W.W. Wu, Ag/AgCl modified self-doped $\mathrm{TiO}_{2}$ hollow sphere with enhanced visible light photocatalytic activity, J. Alloys Compd. 657 (2016) 44-52.

[48] M. Zhu, P. Chen, M. Liu, Highly efficient visible-light-driven plasmonic photocatalysts based on graphene oxide-hybridized one-dimensional Ag/ $\mathrm{AgCl}$ heteroarchitectures, J. Mater. Chem. 22 (2012) 21487-21494. 